\title{
Innate Immunity and Breast Milk
}

\author{
Nicole Theresa Cacho ${ }^{1 *}$ and Robert M. Lawrence ${ }^{2}$ \\ 'Division of Neonatology, Department of Pediatrics, University of Florida, Gainesville, FL, United States, ${ }^{2}$ Division of Pediatric \\ Infectious Disease, Department of Pediatrics, University of Florida, Gainesville, FL, United States
}

Human milk is a dynamic source of nutrients and bioactive factors; unique in providing for the human infant's optimal growth and development. The growing infant's immune system has a number of developmental immune deficiencies placing the infant at increased risk of infection. This review focuses on how human milk directly contributes to the infant's innate immunity. Remarkable new findings clarify the multifunctional nature of human milk bioactive components. New research techniques have expanded our understanding of the potential for human milk's effect on the infant that will never be possible with milk formulas. Human milk microbiome directly shapes the infant's intestinal microbiome, while the human milk oligosaccharides drive the growth of these microbes within the gut. New techniques such as genomics, metabolomics, proteomics, and glycomics are being used to describe this symbiotic relationship. An expanded role for antimicrobial proteins/ peptides within human milk in innate immune protection is described. The unique milieu

OPEN ACCESS

Edited by:

Joseph M. Bliss,

Women \& Infants Hospital of Rhode Island, United States

Reviewed by:

Dina Weilhammer, Lawrence Livermore National Laboratory (DOE), United States Leonardo H. Travassos, Federal University of Rio de Janeiro,

Brazil

${ }^{*}$ Correspondence:

Nicole Theresa Cacho nicole.cacho@peds.ufl.edu

Specialty section: This article was submitted to Microbial Immunology, a section of the journal

Frontiers in Immunology

Received: 30 January 2017 Accepted: 01 May 2017 Published: 29 May 2017

Citation:

Cacho NT and Lawrence RM (2017) Innate Immunity and Breast Milk.

Front. Immunol. 8:584. doi: 10.3389/fimmu.2017.00584 of enhanced immune protection with diminished inflammation results from a complex interaction of anti-inflammatory and antioxidative factors provided by human milk to the intestine. New data support the concept of mucosal-associated lymphoid tissue and its contribution to the cellular content of human milk. Human milk stem cells (hMSCs) have recently been discovered. Their direct role in the infant for repair and regeneration is being investigated. The existence of these hMSCs could prove to be an easily harvested source of multilineage stem cells for the study of cancer and tissue regeneration. As the infant's gastrointestinal tract and immune system develop, there is a comparable transition in human milk over time to provide fewer immune factors and more calories and nutrients for growth. Each of these new findings opens the door to future studies of human milk and its effect on the innate immune system and the developing infant.

Keywords: human milk, breast milk, innate immunity, colostrum, preterm

\section{INTRODUCTION}

The innate immune system is the first line of defense against infection and is activated within minutes, reacting in a nonspecific, preprogrammed, and patterned manner to various infectious or foreign (non-self) stimuli (1). The infant's immune system is immature at birth, and this immaturity is pronounced for the premature infant placing the infant at increased risk of infection (2). Important developmental immune deficiencies at birth include incomplete physical and chemical barriers, poor innate effector cell function, limited and delayed secretory immunoglobulin A (IgA) production, incomplete complement cascade function, and insufficient anti-inflammatory mechanisms of the respiratory and gastrointestinal (GI) tracts.

Human milk is the everchanging secretions of the human breast, an evolving composition of nutrients and active factors. Just as nutrition and protection of the fetus occurs through the mutable 
nature of the uterus, placenta, and amniotic fluid, the evolution of human milk from colostrum through transitional milk to mature milk provides nutrition and protection appropriate for the timeaffected development of the infant (3). There is a large body of evidence documenting the benefits of human breast milk for human infants, in diminishing morbidity and mortality and protecting against specific infections during the period of breastfeeding (4-6). Additional data demonstrate long-term health benefits for the infant (and the mother) beyond the period of lactation $(7,8)$ and led to the current recommendations for duration of exclusive breastfeeding from the American Academy of Pediatrics (9) and the World Health Organization. Although research into the specific factors in human breast milk, which lead to the remarkable health benefits of exclusive breastfeeding, has been ongoing for decades; there are still intriguing mysteries of how human milk contributes to the development and regulation of both the infant's innate (10) and adaptive immune function (11-13).

Elucidating the relationship between the innate immune system and human milk, as well as their individual and interactive transition over time, remains challenging. Many components in milk are multifunctional, serving as enzymes, antimicrobial proteins/peptides (AMPs), growth factors, chemokines, antioxidants, anti-inflammatory elements, prebiotics, probiotics, and nutrients for the growing infant $(14,15)$. The use of modern molecular approaches such as microbial genomics, metabolomics, proteomics, and glycomics has led to novel discoveries in both composition and function of human milk components. This review will focus on the current understanding of the critical interactions between human breast milk and the infant's developing innate immune system (Figure 1) (12, 16-18).

\section{CHEMICAL BARRIERS OF INNATE IMMUNITY}

The chemical barrier of the intestine is predominately the mucus layers lining the GI tract. These mucus layers minimize antigenic contact between epithelial cells and commensal bacteria as well as potentially pathogenic bacteria. Antimicrobial peptides produced by Paneth cells, released into the mucus layer, bolster this chemical barrier innate effect by neutralizing microbes via various mechanisms. The multifunctionality of individual human milk factors adds another layer of complexity to the innate protection effected within the intestinal mucus layers.

Human milk oligosaccharides (HMOs) are the predominant glycans and important nutrients in human milk. They function in direct pathogen binding and as prebiotics facilitating the establishment of a healthy infant microbiome (2). The human milk glycoproteins (HMGPs) vary in size, structure, and amount in human milk and can be classified based on their location relative to the cell (secreted or attached to the cell membrane) and the different mucus layers. Mucin 1 (MUC1) and Mucin 4 (MUC4), gangliosides (GM1, GM3, and GD3), and glycoproteins similarly function by binding pathogens and do so without stimulating an inflammatory response (19). MUC1 and MUC4 have been described as binding to specific pathogens including HIV, rotavirus, Escherichia coli, and Salmonella. Other HMGPs have demonstrated binding to H. influenzae, Streptococci, Helicobacter pylori, Reovirus, E. coli, and Burkholderia cepacia. The specific molecular mechanism of this binding by glycans and interference with infection by the pathogen requires additional clarification. Unraveling the complex glycoprotein-ligand interactions will require application of newer technologies such as nanosurface plasmon resonance and glycan microarrays (20). Another challenge is to demonstrate whether, how, and when this occurs in a mother-infant dyad exposed to a specific pathogen through changes in the glycan composition of the mother's milk (21).

Lactoferrin (LF), another glycoprotein abundant in colostrum and transitional milk and ubiquitously expressed in most exocrine secretions, is one of the best studied glycoproteins in human milk. LF has multiple functions in host defense through binding iron, binding to bacterial membranes, inhibition of tumor necrosis factor-alpha (TNF- $\alpha$ ) and interleukin-1 $\beta$ (IL-1 $\beta$ ), stimulating the activity, maturation of lymphocytes, and contributing to an anti-oxidizing mileau (22). Peptide breakdown products of LF, lactoferricin and lactoferrampin, have specific direct antibacterial and antifungal effects (23).

There are numerous other HMGPs that have been described but the extent and specificity of their immune function need to be elucidated. Lactadherin can inactivate viruses and limits inflammation by increasing the effective phagocytosis of apoptotic cells. The sialic acid component of lactadherin seems to directly interact with rotavirus while the protein backbone of the molecule demonstrates a proangiogenic effect on neovascularization. The list of bioactive glycoproteins in human milk is still expanding, and their individual multifunctional nature is just being described. Butyrophilin, leptin, adiponectin, bile salt-stimulated lipase lysozyme, lactoperoxidase (LP), xanthine dehydrogenase, $\alpha$-lactalbumin, $\kappa$-casein, and $\beta$-casein are just a few of these glycoproteins requiring additional study (19).

\section{CELLULAR CONTRIBUTORS TO INNATE IMMUNITY}

The cellular layers of the infant's intestine include the barrier of epithelial cells with the tight junctions, specialized goblet, Paneth and microfold cells, the lamina propria and Peyer's patches with macrophages, neutrophils, and dendritic cells. The immunity provided by these cellular interactions is partially innate and partially the beginning of adaptive immunity (24). Immaturity of the intestinal barrier function, limitations in production of AMPs, and ineffective response and action of epithelial cells and phagocytes place the infant at risk for infection in the neonatal period $(25,26)$.

Within fresh human milk, there is a remarkable repertoire of heterogeneous living cells. Cells originating in the breast include lactocytes (secretory cells), myoepithelial cells from the ducts as well as progenitor cells and mammary stem cells, and a small number of squamous epithelial cells from the nipple and skin of the breast. Cells originating from the blood, which are in human milk, include immune cells (macrophages, neutrophils, and lymphocytes), hematopoietic progenitor cells, and hematopoietic stem cells $(27,28)$. The role of live breast cells in human milk in the infant remains uncertain. New theories are being formulated to explain the fact that these breast milk epithelial cells are 


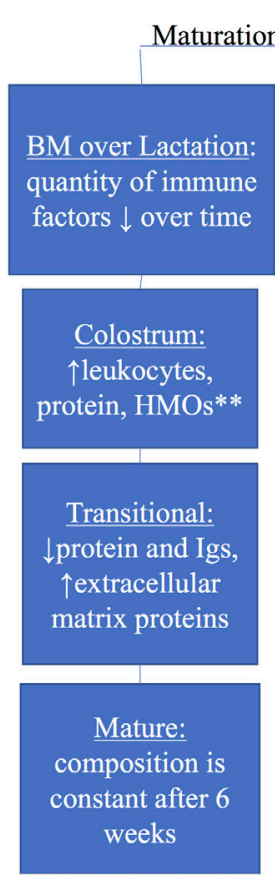

\section{Innate Immunity and BM*}

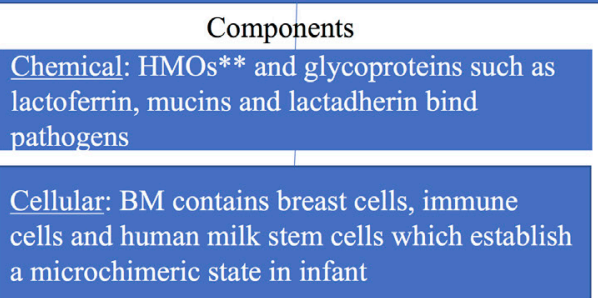

Microbiota: BM directly provides a core yet

personalized set of microbes and prebiotics to

the GI tract which influences immune system

Pathogen Recognition: BM contains sIgA,

TLRs, human $\beta$-defensin- 1 , lactoferrin, soluble

CD14 which function in non-specific binding

Anti-Inflammatory: cytokines, immune/growth

factors, antioxidants in BM balance protective

and destructive effects of inflammation
Gestation

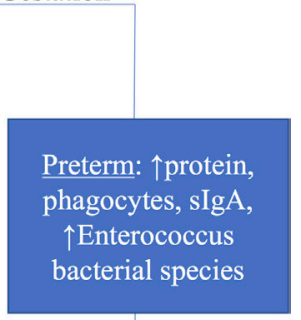

Term: improved

function of

neutrophils,

$\uparrow$ passively acquired

Igs,

$\uparrow$ Bifidobacterium

bacterial species

*Breast Milk

**Human Milk

Oligosaccharides

FIGURE 1 | Summary of innate immunity and breast milk with groups including components of the innate immunity, maturation of breast milk over lactation, and breast milk by gestation (preterm and term).

capable of motility and can in primary culture form functioning mammospheres $(29,30)$. Hassiotou et al. (29) explain the fact that these cells are in different stages of differentiation as a continuum of mammary development, albeit that does not answer the question why they are in such large numbers in expressed human breast milk.

There are large numbers of macrophages present in early lactation that decrease with the maturation of the milk $(31,32)$. These macrophages appear to function by phagocytizing pathogens without initiating a significant, unregulated inflammatory response. An increase in milk leukocytes, above the number usually present at the specific stage of lactation, occurs with infection in either the mother or the infant and suggests a functional role for milk leukocytes in protecting the infant $(21,33,34)$. Breast milk from mothers of infants with severe bronchiolitis demonstrated an increased number of live cells. Those live cells produced a specific cytokine profile response when stimulated with live respiratory syncytial virus, a common cause of bronchiolitis (35). These data directly support the concept of mucosal-associated lymphoid tissue and that cells and/or activating factors are transferred from the mother in her breast milk to the infant at the time of infection or exposure to an infection. Additional studies measuring changes in the bioactive factors in human milk of mothers with sick infants or who are sick themselves for various specific infections should provide more insight into the essential bioactive factors in human milk for protection against specific infections.

The human milk stem cells (hMSCs) likely play an important role in the "regeneration" of the breast in preparation for lactation and theoretically in the infant's tolerance of maternal cell antigens as these cells from human milk do establish a microchimeric state in the infant. Hassiotou et al. (29) have demonstrated the pleuripotential differentiation nature of hMSCs. hMSCs were able to differentiate into the three germ layer cell lineages, in in vitro testing. Expression of octamer-binding transcription factor 4, related to "self-renewal" functions, is upregulated in hMSCs isolated from human milk (33). Using milk from genetically modified mice, Hassiotou et al. (36) demonstrated the persistence of modified milk stem cells within the brain, thymus, pancreas, liver, spleen, and kidneys of non-modified mice (36). These data specifically suggest a potential role for hMSCs in tissue regeneration in the breastfed infant and perhaps regeneration of cells of the infant's innate immune system. Beyond that, the hMSCs in human milk may provide a ready source of patient-specific stem cells with a true multilineage potential for the study of such stem cells and variables related to breast cancer, tissue regeneration, and even bioengineering.

\section{MICROBIOTA}

In the past, human milk was considered sterile, but that is far from the truth. Using culture techniques, the majority of bacteria identified as facultative anaerobes in human milk belong to the Staphylococcus and Streptococcus species and other species in smaller numbers (Propionibacterium, Rothia, Enterococcus, and Lactobacillus species) $(37,38)$. Obligate anaerobic bacteria were later identified, Bifidobacterium and Bacteroides species (39-41). Culture-independent techniques (sequencing and metagenomics analysis) have demonstrated a significantly more complex and diverse group of bacteria in human milk $(42,43)$. There is a large 
degree of interindividual variability in the milk microbiome. Similar factors affecting the infant's or the mother's intestinal microbiomes also affect the milk microbiome (genetics, mode of delivery, geographic area, gestational age, maternal diet and nutrition, antibiotics, lactation stage, etc.) $(42,44)$. Nevertheless, most samples from healthy women appear to contain a "core" microbiome $(34,45)$. Hunt et al. name nine bacterial groups and Jimenez et al. reported seven bacterial groups. These two analyses shared just three common bacterial groups: Staphylococcus, Streptococcus, and Propionibacterium $(34,45)$. Variability between these two studies and others (43) can be attributed to the use of different primers, types of sequencing, comparison with different microbial reference libraries, possible variation due to geographic regions, and timing of the milk collection as well as technique and sterility of milk collection $(43,46,47)$.

The actual origin of the milk microbiota remains uncertain (48). Postulated origins include mammary gland itself, skin flora of the breast, an "entero-mammary pathway" where intestinal bacteria translocate and home to the breast and retrograde flow from the infant's mouth into the breast $(12,49-51)$. Tracing the origins of milk microbiota and the relative influence of environmental factors on its makeup should inform our understanding of the role of the common groups of bacteria or their interaction in symbiosis with the infant's innate immune system and developing intestine.

Human milk directly contributes to the establishment of the intestinal microbiota and facilitates a symbiosis between that microbiota and the infant by providing essential nutrients, in particular milk glycans or HMOs, for microbial metabolism (52). Nanthakumar et al. (53) proposed that specific microbiota colonizing the gut early on in the infant induce the expression of intestinal epithelial cell (IEC) fucosyltransferase 2. This leads to fucosylation of surface markers on GI epithelial cells, which enhances the growth of microbes utilizing fucose as part of their metabolism (53). Microorganisms utilize the available glycosaminoglycans, glycoproteins, glycolipids, and oligosaccharides (milk glycans) as prebiotics to facilitate growth (54). The intestinal microbiota limits the growth of pathogenic bacteria by competition for nutrients and receptors. Specific microbes facilitate the formation of the intestinal mucus layer and the development of the IEC barrier and submucosal lymphoid structures. Separately, the HMOs bind to surface molecules of bacteria and viruses preventing binding to the intestinal epithelium and appear to diminish intestinal inflammation via signaling pathways (54). Newburg and Morelli (55) describe the symbiosis of the microbiota and the infant's intestinal development as dependent on HMOs in the mother's milk. The "commensal" microbes, specifically Bacteroides and Bifidobacterium, induce mucosal glycan production, which further supports microbial growth, and the microbes convert indigestible milk glycans to absorbable short-chain fatty acids (55). He et al. (56) describe two different HMOs ( $3^{\prime}$-galactosyllactose and $2^{\prime}$-fucosyllactose) present in high amounts in colostrum. These HMOs affect pathogen-associated molecular pattern signaling pathways [Toll-like receptor (TLR) 3, TLR5, IL-1 $\beta$, or cluster of differentiation 14 (CD14) expression and binding] decreasing cytokine production and the inflammatory response $(56,57)$. There are now even clinical trials examining the effect of probiotics added to the diet of lactating women, which increased the levels of secretory immunoglobulin A (sIgA) in the infant's stool and on IL-6 mean values in colostrum and on IL-10 and TGF- $\beta 1$ mean values in mature breast milk. This demonstrates the possibility of potentially beneficial microbes influencing the content of the woman's breast milk and subsequently the infants' GI tract via specific bioactive factors reaching the infant. The ultimate benefit to the infant remains to be studied (58). Human milk is not simply adding additional bacteria to the infant's gut and intestinal microbiome but providing both bacteria and prebiotics to function in a symbiotic relationship creating the milieu in which the infant's innate intestinal immunity functions and the intestine develops. How that symbiotic interaction influences the infant's health in the future, as it relates to immune protection and immune reactivity (allergy or autoimmune disease) will require careful research involving not only genomics, metabolomics, proteomics, and glycomics but also epigenetics and techniques still need to be developed.

\section{INNATE MECHANISMS OF PATHOGEN RECOGNITION}

There are specific TLRs present in human milk, including TLR2, TLR3, TLR5 as well as soluble CD14 (sCD14), and human $\beta$-defensin-1 (hBD-1), which function as pattern recognition receptors (PRRs) and AMPs (59). Chatterton et al. (59) discuss the potential role of PRRs in human milk affecting the protective response in the intestine balanced with other bioactive factors in milk affecting an anti-inflammatory mileau. There is evidence that TLR responses in the infant are modified by soluble TLRs (sTLRs) and SCD14. The interaction of both sTLRs and sCD14 with other bioactive factors in human milk upregulates and downregulates the action of various TLR-mediated inflammatory responses $(60,61)$. LeBouder et al. (61) demonstrated the effect of human milk on TLR-mediated microbial recognition. They describe specific responses on epithelial cells, monocytes, dendritic cells, and peripheral blood monocytic cells. The responses were different based on which TLRs were activated. Infant formulas did not exhibit such effects.

Immunoglobulins are the most recognized immune protective component in human breast milk. As preformed Igs from the mother, they constitute a discrete group of proteins capable of pathogen recognition. sIgA is the principal Ig in human milk ( $>90 \%$ of the Ig fraction), immunoglobulin M (IgM) in the pentameric form is next most abundant. There is a small amount of immunoglobulin G (IgG) in colostrum and transitional milk, with IgG becoming a much larger proportion of human milk Igs in mature milk (62). Secretory IgA binds pathogens blocking infection without stimulating a significant inflammatory response. In a largely innate immune-like action, sIgA simply blocks the pathogens contact with the intestinal epithelial layer and traps the pathogens within the mucin layers. The action of sIgA in the extracellular space is different from sIgA's intracellular neutralization of viruses and bacterial lipopolysaccharides within epithelial cells. The glycan sugar component (galactose, fucose, and mannose) of sIgA contributes to sIgA resistance to proteolysis in the intestine and functions through a broad spectrum of 
binding of pathogenic bacteria when compared with the antigen specific binding of the variable region of the Ig structure. The broad spectrum binding related to the glycan sugar component of sIgA is more consistent with an innate immune response which would explain the absence of an increase in specific sIgA in the milk of mothers with sick infants (21).

Immunoglobulin $\mathrm{M}$ causes agglutination of recognized pathogens and complement activation as well as innate immune-like activities. Immunoglobulin $\mathrm{G}$ ( $\mathrm{IgG}$ ) activates phagocytosis with antigen transport to the lamina propria for B-cell activation affecting the infant's adaptive response. The list of pathogens (viruses, bacteria, fungi, and parasites) recognized by human milk Igs is extensive (63). Gao et al. (64) report data from proteomic analysis of human milk, which demonstrate increased amounts of sIgA and IgM in transitional milk with IgG predominating in mature milk. They suggest that this transition fits with the infant's developing immunity and evolving adaptive immunity to produce increasing amounts of IgG.

There are various other AMPs, in human milk, active in microbe killing, which can supplement the protection of the immature neonatal intestine $(14,65)$. hBD-1 is one example of an AMP in human milk, which affects pathogen membrane permeability and cytokine stimulation in the intestine (66). Other proteins [lysozyme, LF (and peptide derivatives of it-lactoferricin and lactoferrampin), $\alpha$-lactalbumin, transferring, and osteopontin $(\mathrm{OPN})$ ] within human milk are recognized as important AMPs functioning via various mechanisms some of which enhance the anti-inflammatory effects of human milk (59, 64, 67). LF and its derivatives demonstrate a wide variety of actions on various targets including iron deprivation, destabilization of microbial membranes, binding microbial receptors, affecting chemokine production, stimulating epithelial cell growth, stimulating T-cell growth and differentiation, and production of reactive oxygen species (ROSs). LF also interacts with other components in human milk such as OPN, ceruloplasmin, and neutrophil peroxidase, although the exact significance and function of these interactions remain uncertain $(22,68)$. Xanthine oxidoreductase, another protein found in large amounts in milk and upregulated in mature milk, affects mammary epithelium, generation of milk fat droplet membranes, and adds to the bactericidal effect of human milk by synthesizing ROS $(64,66)$. These different AMPs do not simply act in microbial recognition and inactivation; each have different secondary functions within the intestine. Equally important is the limited inflammatory response generated by these AMPs in the gut.

\section{ANTI-INFLAMMATORY FACTORS AND EFFECTS}

Maintenance of a homeostasis between protective inflammation and modulation of inflammation is essential to protecting the infant against infection at the same time as limiting the tissue damage due to inflammation (59). Oxidative stress through the production of free radicals does have some potentially beneficial effects for the host in terms of antibacterial action, immune defense, and signal transduction. The oxidative activity within the infant must be maintained in equilibrium with antioxidant capacity of tissues (69). Enterocytes and immune cells produce anti-inflammatory cytokines including transforming growth factor-beta (TGF- $\beta$ ), IL-10, IL-11, and IL-13. These factors act in an innate manner in the intestine.

Human milk caseins, LF, LP, OPN, Igs, superoxide dismutase (SOD), platelet-activating factor acetylhydrolase, and alkaline phosphatase each have both infection protective and anti-inflammatory effects. Specific hormones or growth factors predominantly exert their anti-inflammatory effects on intestinal innate immunity through their action on the proliferation and differentiation of IECs and immune cells (lymphocytes and macrophages) and modulating the inflammatory cytokine response. Transforming growth factors- $\beta 2$ and $-\beta 1$ upregulate tight junction proteins (caludin-1 and claudin-4) and downregulate TNF- $\alpha$ and IL- $1 \beta$. In addition to growth stimulation, TGF- $\beta$ has anti-inflammatory properties through stimulation of epithelial cell migration and repair of the epithelium after mucosal damage (70). Similarly, insulin-like growth factors, milk fat globule epidermal growth factor-8 (MFG-E8), and epidermal growth factor (EGF) influence growth and proliferation of IECs. Both MFG-E8 and EGF diminish the activation of nuclear factor kappa-lightchain enhancer of activated B cells (59). Trefoil factor 3 (TFF3) is an effector molecule that is present in the intestine and in large amounts in human breast milk. Generally, this molecule improves healing in the GI tract. The TFF3 present in breast milk produces downregulation of cytokines and promotes hBDs expression in IECs (71). Glucagon-like peptide 1 (GLP-1) is secreted from the enteroendocrine cell in the distal intestine and plays a role in regulating glucose metabolism and food intake. GLP-1 likely acts through vagal afferent pathway ultimately influencing feeding behavior (72). Recently, the first study to report GLP-1 in human milk showed that it was higher in hindmilk compared to foremilk and was correlated with infant weight gain during the first 6 months of life (73). Alternative forms of neonatal nutrition such as formula and TPN do not contain these anti-inflammatory properties, which may put these infants at a disadvantage by creating a relative deficiency of anti-inflammatory factors and activity.

Any enteral feeding directly stimulates growth of the neonatal gut. Human milk contains specific direct growth factors including platelet-derived growth factor, hepatocyte growth factor (HGF), vascular endothelial growth factor, and insulin (74-76). Each of these is important in angiogenesis, cell development, and tissue proliferation. HGF is expressed in the intestinal tissues and is present in human milk. HGF may play a role in mucosal growth and repair (77). Animal studies have shown that HGF given after intestinal resection or colitis has improved gut proliferation and nutrient transport $(78,79)$. Weiss et al. (80) describe declining levels of anti-inflammatory, proresolving lipoxin A4 (LXA4), and resolvin D1 (RvD1) and D2 (RvD2) in the lipid profile of human milk from colostrum through the first month of life. The average amount of LXA4 in human milk was two times the amount of proinflammatory leukotriene B4 (LTB4) (80). This highlights the importance of minimizing inflammation in the early period of an infant's life.

Specific antioxidants in human milk include vitamins A, E, C, LF, lysozyme, glutathione peroxidase, SOD, catalase, 
ceruloplasmin, coenzyme Q10, thioredoxin, leptin, adiponectin, and trace elements-iron, copper, zinc, and selenium (69). These antioxidants act by reacting directly with a free radical before damage occurs or by interfering with the ongoing oxidation in liquid phase or in cell membranes. The total antioxidative capacity of human milk is highest in colostrum and declines over lactation with variability from person to person and time to time (69). Adiponectin, leptin, LF, and lysozyme each have antioxidant effects, and their concentrations in human milk also vary over time (81). Vitamin A or its derivatives bind to radicals of oxygen, thiol, or peroxide, limiting their oxidative damage on cells. There are higher concentrations of vitamin A in colostrum than mature milk (82). Higher concentrations of $\alpha$-tocopherol (the predominant form of vitamin E) are in colostrum than transitional or mature milk (83). Vitamin E forms part of the milk fat globule membrane and constitutes the major portion of antioxidative function of breast milk at 1 month of age (84). Vitamin C is a hydrosoluble vitamin in human milk and an effective antioxidant in extracellular fluids.

Nucleotides, nucleosides, and nucleic acids are essential to cellular metabolic function. They constitute approximately $15-20 \%$ of the non-protein nitrogen or total potentially available nucleosides in human milk (85). Nucleotides function predominantly in cellular energy metabolism related to adenosine triphosphate, as messengers and coenzymes in metabolic pathways, and in nucleic acid production and salvage. In the face of infection, nucleotides are essential to the immune response (cellular activation, proliferation and action, and cellular signal transduction) and the repair of intestinal inflammation and damage. Brunser et al. (86) reported on the benefit of nucleotide supplementation for infants less than 6 months old at decreasing the severity and incidence of diarrhea. They postulated that the effect was due to effects on the intestinal integrity and repair as an example of an anti-inflammatory effect.

\section{EVOLUTION OF BIOACTIVE FACTORS IN HUMAN MILK OVER LACTATION}

The composition of human milk is dynamic with significant change from colostrum, transitional to mature milk, between preterm and term milk and with interindividual and intraindividual variation. Specifically, HMOs show interindividual variation relative to the total number of HMOs and individual HMOs varying with mother's Lewis blood group and secretor status.

Colostrum is produced between birth through the first 5 days of lactation, transitional milk is from 5 days to 2 weeks postpartum and maturation of the milk continues until it is "fully mature" at 4-6 weeks postpartum. There is only a small volume of colostrum produced, rich in leukocytes, protein, HMOs, and bioactive factors-IgA, LF, EGF, TGF- $\beta$, colony-stimulating growth factor, and antioxidants (31). Transitional milk has decreasing amounts of protein and Igs and increasing lactose and fat and water-soluble vitamins resulting in a higher caloric density of the milk to meet the infant's growth demands while the quantities of bioactive factors declines over time. The composition of mature milk remains constant after 6 weeks through the remainder of the lactation period. The amount of Igs and LF in milk decreases over the first
3-4 months, while the amount of lysozyme increases (87). Tregoat et al. (88) described declining amounts of mannan-binding lectin concentrations in transition from colostrum to mature milk. More recent published studies using proteomic analysis of human milk $(16,64,89)$ continue to facilitate our understanding of the complex nature of human milk and its role related to immune function and intestinal development. Gao et al. (64) describe a similar transition of the various Igs, from colostrum to mature milk using proteomic analysis of the milk. They describe a large percentage of milk proteins having to do with immune function including complement factors and serine protease inhibitors important in regulating the complement system. The quantities of these factors declined in transition from colostrum to mature milk. They also identified proteins associated with the extracellular matrix including cytokines, fibronectin, tenascin, and OPN. These proteins were more prevalent in transitional milk. Glutathione and antioxidant activity-related proteins were more common in mature milk. Overall, the quantity of immune factors and immune effects of human milk diminish over time in parallel with the developing immune system of the infant. Nevertheless, it will be essential to understand the specific roles of the various bioactive components of human milk and how the change in milk composition over time influences the evolving effects of human milk on the intestine and innate immunity.

\section{DIFFERENCES IN PRETERM AND TERM HUMAN MILK}

Preterm babies require additional nutrition and immune protection compared to term infants. Interestingly, preterm breast milk has been found to contain increased nutrients such as protein (90) and higher concentrations of certain immune factors. Preterm human milk also has higher amounts of phagocytes and secretory IgA (24). These increased amounts may serve a protective role since premature infants have poorly functioning neutrophils, limited production of Igs, and lower levels of passively acquired Igs.

A few studies reported a difference in breast milk microbiome when comparing preterm and term milk. Some trends include more Bifidobacterium in term milk (91) and more Enterococcus in preterm milk (91). A study looking at preterm infants, testing stool and breast milk samples, found a high proportion of antibiotic-resistant high-risk clones in both fecal and milk samples during the neonatal intensive care unit (NICU) admission (92). Differences are also seen in gut microbiome between term and preterm infants. Variations in microbiota of preterm infants have been described as predisposing to development of necrotizing enterocolitis (NEC) (93-97). Hormones and cytokines also vary by gestational age. EGF has anti-inflammatory properties and is higher in preterm milk compared to full-term milk (24) The cytokine IL-10, with anti-inflammatory properties, was detected in lower amounts in breast milk for infants with increased risk of NEC (98). Trend et al. reported that TGF- $\beta 2$ concentrations in human milk were significantly higher in the extreme premature infant group compared to the term infant group (99). Castellote et al. also reported that TGF- $\beta 2$ was higher in preterm infants compared with term infants (100). A study by Maheshwari et al. 
demonstrated that this anti-inflammatory cytokine suppresses endotoxin-induced cytokine responses of gut macrophages in the preterm infant in vitro and protects rat pups from gut injury in vivo (101). They also reported that percentages of IL-10 and TNF- $\alpha$ were lower in preterm milk compared to term milk.

Trend et al. found that AMPs can limit the in vitro growth of bacteria associated with neonatal sepsis (52). This is particularly important in preterm infants who are at increased risk of late-onset sepsis. The hBD-1 was higher in preterm colostrum compared to term colostrum (99). Wang et al. (102) also found that hBD-1 and hBD-2 were in higher concentration in mature preterm milk than mature term milk. Armogida et al. and Trend et al. both found that human $\alpha$-defensin 5 levels were not affected by preterm birth, which suggests that these defensins are differentially regulated $(99,103)$. The amount of LF in human milk does not seem to be affected by gestational age (99) but is more dependent on milk volume expressed. There are conflicting results for concentrations of lysozyme in preterm and term milk.

It has been proposed that the increased levels of immune factors in preterm milk may be a result of a compensatory mechanism whereby in the mother during preterm labor, the breast shifts the immune content of the milk to provide more protection. Another explanation suggested by Goldman et al. (62) is that increased immune factors in preterm milk may be due to increased maternal systemic inflammation, a postulated condition leading to preterm delivery. The mechanisms for the differences between preterm

\section{REFERENCES}

1. Medzhitov R, Janeway C Jr. Innate immunity. N Engl J Med (2000) 343(5): 338-44. doi:10.1056/NEJM200008033430506

2. Newburg DS, Walker WA. Protection of the neonate by the innate immune system of developing gut and of human milk. Pediatr Res (2007) 61(1):2-8. doi:10.1203/01.pdr.0000250274.68571.18

3. Andreas NJ, Kampmann B, Mehring Le-Doare K. Human breast milk: a review on its composition and bioactivity. Early Hum Dev (2015) 91(11):629-35. doi:10.1016/j.earlhumdev.2015.08.013

4. Bahl R, Frost C, Kirkwood BR, Edmond K, Martines J, Bhandari N, et al. Infant feeding patterns and risks of death and hospitalization in the first half of infancy: multicentre cohort study. Bull World Health Organ (2005) 83(6):418-26. doi:10.1590/S0042-96862005000600009

5. Duijts L, Jaddoe VW, Hofman A, Moll HA. Prolonged and exclusive breastfeeding reduces the risk of infectious diseases in infancy. Pediatrics (2010) 126(1):e18-25. doi:10.1542/peds.2008-3256

6. Edmond KM, Zandoh C, Quigley MA, Amenga-Etego S, Owusu-Agyei S, Kirkwood BR. Delayed breastfeeding initiation increases risk of neonatal mortality. Pediatrics (2006) 117(3):e380-6. doi:10.1542/peds.2005-1496

7. Ip S, Chung M, Raman G, Chew P, Magula N, DeVine D, et al. Breastfeeding and maternal and infant health outcomes in developed countries. Evid Rep Technol Assess (Full Rep) (2007) 153:1-186.

8. Kramer MS, Kakuma R. Optimal duration of exclusive breastfeeding. Cochrane Database Syst Rev (2012) 8:CD003517. doi:10.1002/14651858. CD003517.pub2

9. Gartner LM, Morton J, Lawrence RA, Naylor AJ, O’Hare D, Schanler RJ, et al. Breastfeeding and the use of human milk. Pediatrics (2012) 129:e827-41. doi:10.1542/peds.2011-3552

10. Iwasaki A, Medzhitov R. Control of adaptive immunity by the innate immune system. Nat Immunol (2015) 16(4):343-53. doi:10.1038/ni.3123

11. Pacheco AR, Barile D, Underwood MA, Mills DA. The impact of the milk glycobiome on the neonate gut microbiota. Annu Rev Anim Biosci (2015) 3:419-45. doi:10.1146/annurev-animal-022114-111112 and term milk remain unknown. Interestingly, most immune factors decrease over the first month regardless of gestational age, thus term and preterm milk become more similar over time as the chronological age of the baby increases.

\section{SUMMARY}

Feeding an infant human breast milk is not a matter of filling the infant with an "appropriate" amount of important nutrients and a protective level of bioactive factors. Although the various factors do complement and supplement the innate immunity of the infant, they actively affect the ongoing development of the infant's immunity and intestinal development. As Lars Bode et al. declared, "Human milk is alive, ..." (27). The cells, the microbes, and the bioactive factors make milk alive, and the interactions of human milk with its natural host, the infant, create a symbiotic commensal relationship. This is the challenge to explain and understand the complexity and dynamic relationship between the everchanging secretion, human breast milk and the developing, evolving human infant.

\section{AUTHOR CONTRIBUTIONS}

All authors have made substantial, direct, and intellectual contribution to the work and approved it for publication. NC is primarily responsible for Figure 1.
12. McGuire MK, McGuire MA. Human milk: mother nature's prototypical probiotic food? Adv Nutr (2015) 6(1):112-23. doi:10.3945/an.114.007435

13. Alexander KL, Targan SR, Elson CO III. Microbiota activation and regulation of innate and adaptive immunity. Immunol Rev (2014) 260(1):206-20. doi:10.1111/imr.12180

14. Mandal SM, Bharti R, Porto WF, Gauri SS, Mandal M, Franco OL, et al. Identification of multifunctional peptides from human milk. Peptides (2014) 56:84-93. doi:10.1016/j.peptides.2014.03.017

15. Chirico G, Marzollo R, Cortinovis S, Fonte C, Gasparoni A. Anti infective properties of human milk. J Nutr (2008) 138(9):1801S-6S.

16. De Leoz ML, Kalanetra KM, Bokulich NA, Strum JS, Underwood MA, German JB, et al. Human milk glycomics and gut microbial genomics in infant feces show a correlation between human milk oligosaccharides and gut microbiota: a proof-of-concept study. J Proteome Res (2015) 14(1):491-502. doi:10.1021/pr500759e

17. Qian L, Zhao A, Zhang Y, Chen T, Zeisel SH, Jia W, et al. Metabolomic approaches to explore chemical diversity of human breast-milk, formula milk and bovine milk. Int J Mol Sci (2016) 17(12):E2128. doi:10.3390/ ijms 17122128

18. Roncada P, Stipetic LH, Bonizzi L, Burchmore RJ, Kennedy MW. Proteomics as a tool to explore human milk in health and disease. J Proteomics (2013) 88:47-57. doi:10.1016/j.jprot.2013.04.008

19. Liu B, Newburg DS. Human milk glycoproteins protect infants against human pathogens. Breastfeed Med (2013) 8(4):354-62. doi:10.1089/bfm.2013.0016

20. Reuel NF, Mu B, Zhang J, Hinckley A, Strano MS. Nanoengineered glycan sensors enabling native glycoprofiling for medicinal applications: towards profiling glycoproteins without labeling or liberation steps. Chem Soc Rev (2012) 41(17):5744-79. doi:10.1039/c2cs35142k

21. Riskin A, Almog M, Peri R, Halasz K, Srugo I, Kessel A. Changes in immunomodulatory constituents of human milk in response to active infection in the nursing infant. Pediatr Res (2012) 71(2):220-5. doi:10.1038/ pr.2011.34

22. Legrand D. Overview of lactoferrin as a natural immune modulator. J Pediatr (2016) 173(Suppl):S10-5. doi:10.1016/j.jpeds.2016.02.071 
23. Yin C, Wong JH, Ng TB. Recent studies on the antimicrobial peptides lactoferricin and lactoferrampin. Curr Mol Med (2014) 14(9):1139-54. doi:10.2174/1566524014666141015151749

24. Jakaitis BM, Denning PW. Human breast milk and the gastrointestinal innate immune system. Clin Perinatol (2014) 41(2):423-35. doi:10.1016/j. clp.2014.02.011

25. Anderson RC, Dalziel JE, Gopal PK, Bassett S, Ellis A, Roy NC. The role of intestinal barrier function in early life in the development of colitis. In: Fukata M, editor. Colitis. Rijeka: InTechOpen (2012). p. 3-30.

26. Nussbaum C, Gloning A, Pruenster M, Frommhold D, Bierschenk S, GenzelBoroviczeny $\mathrm{O}$, et al. Neutrophil and endothelial adhesive function during human fetal ontogeny. JLeukoc Biol (2013) 93(2):175-84. doi:10.1189/ jlb.0912468

27. Bode L, McGuire M, Rodriguez JM, Geddes DT, Hassiotou F, Hartmann PE, et al. It's alive: microbes and cells in human milk and their potential benefits to mother and infant. Adv Nutr (2014) 5(5):571-3. doi:10.3945/an.114. 006643

28. Hassiotou F, Geddes D. Anatomy of the human mammary gland: current status of knowledge. Clin Anat (2013) 26(1):29-48. doi:10.1002/ca.22165

29. Hassiotou F, Beltran A, Chetwynd E, Stuebe AM, Twigger AJ, Metzger P, et al. Breastmilk is a novel source of stem cells with multilineage differentiation potential. Stem Cells (2012) 30(10):2164-74. doi:10.1002/stem.1188

30. Hassiotou F, Geddes DT, Hartmann PE. Cells in human milk: state of the science. J Hum Lact (2013) 29(2):171-82. doi:10.1177/0890334413477242

31. Ballard O, Morrow AL. Human milk composition: nutrients and bioactive factors. Pediatr Clin North Am (2013) 60(1):49-74. doi:10.1016/j. pcl.2012.10.002

32. Hassiotou F, Geddes DT. Immune cell-mediated protection of the mammary gland and the infant during breastfeeding. Adv Nutr (2015) 6(3):267-75. doi:10.3945/an.114.007377

33. Hassiotou F, Hepworth AR, Metzger P, Tat Lai C, Trengove N, Hartmann PE, et al. Maternal and infant infections stimulate a rapid leukocyte response in breastmilk. Clin Transl Immunol (2013) 2(4):e3. doi:10.1038/cti.2013.1

34. Jimenez E, de Andres J, Manrique M, Pareja-Tobes P, Tobes R, Martinez-Blanch JF, et al. Metagenomic analysis of milk of healthy and mastitis-suffering women. J Hum Lact (2015) 31(3):406-15. doi:10.1177/ 0890334415585078

35. Bryan DL, Hart PH, Forsyth KD, Gibson RA. Immunomodulatory constituents of human milk change in response to infant bronchiolitis. Pediatr Allergy Immunol (2007) 18(6):495-502. doi:10.1111/j.1399-3038.2007.00565.x

36. Hassiotou F, Heath B, Ocal O, Filgueira L, Geddes DT, Hartmann PE, et al. Breastmilk stem cells transfer from mother to neonatal organs. FASEB $J$ (2014) 28(1):Suppl 216.4. Exp Biol (2014), San Diego, CA.

37. Heikkila MP, Saris PE. Inhibition of Staphylococcus aureus by the commensal bacteria of human milk. J Appl Microbiol (2003) 95(3):471-8. doi:10.1046/j.1365-2672.2003.02002.x

38. Solis G, de Los Reyes-Gavilan CG, Fernandez N, Margolles A, Gueimonde M. Establishment and development of lactic acid bacteria and bifidobacteria microbiota in breast-milk and the infant gut. Anaerobe (2010) 16(3):307-10. doi:10.1016/j.anaerobe.2010.02.004

39. Arboleya S, Ruas-Madiedo P, Margolles A, Solis G, Salminen S, de Los ReyesGavilan CG, et al. Characterization and in vitro properties of potentially probiotic Bifidobacterium strains isolated from breast-milk. Int J Food Microbiol (2011) 149(1):28-36. doi:10.1016/j.ijfoodmicro.2010.10.036

40. Martin V, Maldonado-Barragan A, Moles L, Rodriguez-Banos M, Campo RD, Fernandez L, et al. Sharing of bacterial strains between breast milk and infant feces. J Hum Lact (2012) 28(1):36-44. doi:10.1177/0890334411424729

41. Jost T, Lacroix C, Braegger C, Chassard C. Assessment of bacterial diversity in breast milk using culture-dependent and culture-independent approaches. Br J Nutr (2013) 110(7):1253-62. doi:10.1017/S0007114513000597

42. Gomez-Gallego C, Garcia-Mantrana I, Salminen S, Collado MC. The human milk microbiome and factors influencing its composition and activity. Semin Fetal Neonatal Med (2016) 21(6):400-5. doi:10.1016/j.siny.2016.05.003

43. Fitzstevens JL, Smith KC, Hagadorn JI, Caimano MJ, Matson AP, Brownell EA. Systematic review of the human milk microbiota. Nutr Clin Pract (2016) 1-11. doi:10.1177/0884533616670150

44. Cacho N, Neu J. Manipulation of the intestinal microbiome in newborn infants. Adv Nutr (2014) 5(1):114-8. doi:10.3945/an.113.004820
45. Hunt KM, Foster JA, Forney LJ, Schutte UM, Beck DL, Abdo Z, et al. Characterization of the diversity and temporal stability of bacterial communities in human milk. PLoS One (2011) 6(6):e21313. doi:10.1371/journal. pone. 0021313

46. Walker AW, Martin JC, Scott P, Parkhill J, Flint HJ, Scott KP. 16S rRNA genebased profiling of the human infant gut microbiota is strongly influenced by sample processing and PCR primer choice. Microbiome (2015) 3:26. doi:10.1186/s40168-015-0087-4

47. Backhed F, Roswall J, Peng Y, Feng Q, Jia H, Kovatcheva-Datchary P, et al. Dynamics and stabilization of the human gut microbiome during the first year of life. Cell Host Microbe (2015) 17(5):690-703. doi:10.1016/j.chom. 2015.05.012

48. Fernandez L, Langa S, Martin V, Maldonado A, Jimenez E, Martin R, et al. The human milk microbiota: origin and potential roles in health and disease. Pharmacol Res (2013) 69(1):1-10. doi:10.1016/j.phrs.2012.09.001

49. Rautava S. Early microbial contact, the breast milk microbiome and child health. JDev Orig Health Dis (2016) 7(1):5-14. doi:10.1017/ S2040174415001233

50. Perez PF, Dore J, Leclerc M, Levenez F, Benyacoub J, Serrant P, et al. Bacterial imprinting of the neonatal immune system: lessons from maternal cells? Pediatrics (2007) 119(3):e724-32. doi:10.1542/peds.2006-1649

51. McGuire MK, McGuire MA. Got bacteria? The astounding, yet not-so-surprising, microbiome of human milk. Curr Opin Biotechnol (2016) 44:63-8. doi:10.1016/j.copbio.2016.11.013

52. Trend S, Strunk T, Hibbert J, Kok CH, Zhang G, Doherty DA, et al. Antimicrobial protein and peptide concentrations and activity in human breast milk consumed by preterm infants at risk of late-onset neonatal sepsis. PLoS One (2015) 10(2):e0117038. doi:10.1371/journal.pone.0117038

53. Nanthakumar NN, Meng D, Newburg DS. Glucocorticoids and microbiota regulate ontogeny of intestinal fucosyltransferase 2 requisite for gut homeostasis. Glycobiology (2013) 23(10):1131-41. doi:10.1093/glycob/cwt050

54. Newburg DS, He Y. Neonatal gut microbiota and human milk glycans cooperate to attenuate infection and inflammation. Clin Obstet Gynecol (2015) 58(4):814-26. doi:10.1097/GRF.0000000000000156

55. Newburg DS, Morelli L. Human milk and infant intestinal mucosal glycans guide succession of the neonatal intestinal microbiota. Pediatr Res (2015) 77(1-2):115-20. doi:10.1038/pr.2014.178

56. He Y, Liu S, Leone S, Newburg DS. Human colostrum oligosaccharides modulate major immunologic pathways of immature human intestine. Mucosal Immunol (2014) 7(6):1326-39. doi:10.1038/mi.2014.20

57. He Y, Liu S, Kling DE, Leone S, Lawlor NT, Huang Y, et al. The human milk oligosaccharide 2'-fucosyllactose modulates CD14 expression in human enterocytes, thereby attenuating LPS-induced inflammation. Gut (2016) 65(1):33-46. doi:10.1136/gutjnl-2014-307544

58. Baldassarre ME, Di Mauro A, Mastromarino P, Fanelli M, Martinelli D, Urbano F, et al. Administration of a multi-strain probiotic product to women in the perinatal period differentially affects the breast milk cytokine profile and may have beneficial effects on neonatal gastrointestinal functional symptoms. A randomized clinical trial. Nutrients (2016) 8(11):E677. doi:10.3390/ nu8110677

59. Chatterton DE, Nguyen DN, Bering SB, Sangild PT. Anti-inflammatory mechanisms of bioactive milk proteins in the intestine of newborns. Int J Biochem Cell Biol (2013) 45(8):1730-47. doi:10.1016/j.biocel.2013. 04.028

60. He Y, Lawlor NT, Newburg DS. Human milk components modulate toll-like receptor-mediated inflammation. Adv Nutr (2016) 7(1):102-11. doi:10.3945/ an. 115.010090

61. LeBouder E, Rey-Nores JE, Raby AC, Affolter M, Vidal K, Thornton CA, et al. Modulation of neonatal microbial recognition: TLR-mediated innate immune responses are specifically and differentially modulated by human milk. J Immunol (2006) 176(6):3742-52. doi:10.4049/jimmunol.176.6.3742

62. Goldman AS, Garza C, Nichols BL, Goldblum RM. Immunologic factors in human milk during the first year of lactation. J Pediatr (1982) 100(4):563-7. doi:10.1016/S0022-3476(82)80753-1

63. Lawrence RA, Lawrence RM. Chapter 5: Host-resistance factors and immunologic significance of human milk. In: Lawrence RA, Lawrence RM, editors. Breastfeeding: A Guide for the Medical Profession. Philadelphia, PA: Elsevier (2015). p. 146-93. 
64. Gao X, McMahon RJ, Woo JG, Davidson BS, Morrow AL, Zhang Q. Temporal changes in milk proteomes reveal developing milk functions. J Proteome Res (2012) 11(7):3897-907. doi:10.1021/pr3004002

65. Phadke SM, Deslouches B, Hileman SE, Montelaro RC, Wiesenfeld HC, Mietzner TA. Antimicrobial peptides in mucosal secretions: the importance of local secretions in mitigating infection. J Nutr (2005) 135(5):1289-93.

66. Vorbach C, Capecchi MR, Penninger JM. Evolution of the mammary gland from the innate immune system? Bioessays (2006) 28(6):606-16. doi:10.1002/ bies. 20423

67. Wada Y, Lonnerdal B. Bioactive peptides derived from human milk proteins mechanisms of action. J Nutr Biochem (2014) 25(5):503-14. doi:10.1016/j. jnutbio.2013.10.012

68. Sokolov AV, Ageeva KV, Pulina MO, Zakharova ET, Vasilyev VB. Effect of lactoferrin on oxidative features of ceruloplasmin. Biometals (2009) 22(3):521-9. doi:10.1007/s10534-009-9209-4

69. Matos C, Ribeiro M, Guerra A. Breastfeeding: antioxidative properties of breast milk. J Appl Biomed (2015) 13:169-80. doi:10.1016/j.jab.2015.04.003

70. Podolsky DK. Mucosal immunity and inflammation. V. Innate mechanisms of mucosal defense and repair: the best offense is a good defense. Am J Physiol (1999) 277(3 Pt 1):G495-9.

71. Barrera GJ, Tortolero GS. Trefoil factor 3 (TFF3) from human breast milk activates PAR-2 receptors, of the intestinal epithelial cells HT-29, regulating cytokines and defensins. Bratisl Lek Listy (2016) 117(6):332-9. doi:10.4149/ BLL_2016_066

72. Ronveaux CC, Tome D, Raybould HE. Glucagon-like peptide 1 interacts with ghrelin and leptin to regulate glucose metabolism and food intake through vagal afferent neuron signaling. J Nutr (2015) 145(4):672-80. doi:10.3945/ jn.114.206029

73. Schueler J, Alexander B, Hart AM, Austin K, Larson-Meyer DE. Presence and dynamics of leptin, GLP-1, and PYY in human breast milk at early postpartum. Obesity (Silver Spring) (2013) 21(7):1451-8. doi:10.1002/ oby. 20345

74. Kobata R, Tsukahara H, Ohshima Y, Ohta N, Tokuriki S, Tamura S, et al. High levels of growth factors in human breast milk. Early Hum Dev (2008) 84(1):67-9. doi:10.1016/j.earlhumdev.2007.07.005

75. Ozgurtas T, Aydin I, Turan O, Koc E, Hirfanoglu IM, Acikel CH, et al. Vascular endothelial growth factor, basic fibroblast growth factor, insulin-like growth factor-I and platelet-derived growth factor levels in human milk of mothers with term and preterm neonates. Cytokine (2010) 50(2):192-4. doi:10.1016/j. cyto.2010.02.008

76. Patki S, Patki U, Patil R, Indumathi S, Kaingade P, Bulbule A, et al. Comparison of the levels of the growth factors in umbilical cord serum and human milk and its clinical significance. Cytokine (2012) 59(2):305-8. doi:10.1016/j. cyto.2012.04.010

77. Burrin DG, Stoll B. Key nutrients and growth factors for the neonatal gastrointestinal tract. Clin Perinatol (2002) 29(1):65-96. doi:10.1016/ S0095-5108(03)00065-4

78. Schwartz MZ, Kato Y, Yu D, Lukish JR. Growth-factor enhancement of compromised gut function following massive small-bowel resection. Pediatr Surg Int (2000) 16(3):174-5. doi:10.1007/s003830050716

79. Ohda Y, Hori K, Tomita T, Hida N, Kosaka T, Fukuda Y, et al. Effects of hepatocyte growth factor on rat inflammatory bowel disease models. Dig Dis Sci (2005) 50(5):914-21. doi:10.1007/s10620-005-2664-z

80. Weiss GA, Troxler H, Klinke G, Rogler D, Braegger C, Hersberger M. High levels of anti-inflammatory and pro-resolving lipid mediators lipoxins and resolvins and declining docosahexaenoic acid levels in human milk during the first month of lactation. Lipids Health Dis (2013) 12:89. doi:10.1186/1476-511X-12-89

81. Mehta R, Petrova A. Is variation in total antioxidant capacity of human milk associated with levels of bio-active proteins? J Perinatol (2014) 34(3):220-2. doi:10.1038/jp.2013.151

82. Song BJ, Jouni ZE, Ferruzzi MG. Assessment of phytochemical content in human milk during different stages of lactation. Nutrition (2013) 29(1): 195-202. doi:10.1016/j.nut.2012.07.015

83. Szlagatys-Sidorkiewicz A, Zagierski M, Jankowska A, Luczak G, Macur K, Baczek T, et al. Longitudinal study of vitamins A, E and lipid oxidative damage in human milk throughout lactation. Early Hum Dev (2012) 88(6):421-4. doi:10.1016/j.earlhumdev.2011.10.007
84. Tijerina-Saenz A, Innis SM, Kitts DD. Antioxidant capacity of human milk and its association with vitamins $\mathrm{A}$ and $\mathrm{E}$ and fatty acid composition. Acta Paediatr (2009) 98(11):1793-8. doi:10.1111/j.1651-2227.2009.01437.x

85. Leach JL, Baxter JH, Molitor BE, Ramstack MB, Masor ML. Total potentially available nucleosides of human milk by stage of lactation. Am J Clin Nutr (1995) 61(6):1224-30.

86. Brunser O, Espinoza J, Araya M, Cruchet S, Gil A. Effect of dietary nucleotide supplementation on diarrhoeal disease in infants. Acta Paediatr (1994) 83(2):188-91. doi:10.1111/j.1651-2227.1994.tb13048.x

87. Butte NF, Goldblum RM, Fehl LM, Loftin K, Smith EO, Garza C, et al. Daily ingestion of immunologic components in human milk during the first four months of life. Acta Paediatr Scand (1984) 73(3):296-301. doi:10.1111/j. 1651-2227.1994.tb17738.x

88. Tregoat V, Montagne P, Bene MC, Faure G. Changes in the mannan binding lectin (MBL) concentration in human milk during lactation. J Clin Lab Anal (2002) 16(6):304-7. doi:10.1002/jcla.10055

89. van Herwijnen MJ, Zonneveld MI, Goerdayal S, Nolte-'t Hoen EN, Garssen J, Stahl B, et al. Comprehensive proteomic analysis of human milk-derived extracellular vesicles unveils a novel functional proteome distinct from other milk components. Mol Cell Proteomics (2016) 15(11):3412-23. doi:10.1074/mcp.M116.060426

90. Gidrewicz DA, Fenton TR. A systematic review and meta-analysis of the nutrient content of preterm and term breast milk. BMC Pediatr (2014) 14:216. doi:10.1186/1471-2431-14-216

91. Khodayar-Pardo P, Mira-Pascual L, Collado MC, Martinez-Costa C. Impact of lactation stage, gestational age and mode of delivery on breast milk microbiota. J Perinatol (2014) 34(8):599-605. doi:10.1038/jp.2014.47

92. Moles L, Gomez M, Jimenez E, Fernandez L, Bustos G, Chaves F, et al. Preterm infant gut colonization in the neonatal ICU and complete restoration 2 years later. Clin Microbiol Infect (2015) 21(10):936.e1-10. doi:10.1016/j. cmi.2015.06.003

93. Underwood MA, Gaerlan S, De Leoz ML, Dimapasoc L, Kalanetra KM, Lemay DG, et al. Human milk oligosaccharides in premature infants: absorption, excretion, and influence on the intestinal microbiota. Pediatr Res (2015) 78(6):670-7. doi:10.1038/pr.2015.162

94. Stewart CJ, Marrs EC, Nelson A, Lanyon C, Perry JD, Embleton ND, et al. Development of the preterm gut microbiome in twins at risk of necrotising enterocolitis and sepsis. PLoS One (2013) 8(8):e73465. doi:10.1371/journal. pone. 0073465

95. Said MB, Hays S, Maucort-Boulch D, Oulmaati A, Hantova S, Loys CM, et al. Gut microbiota in preterm infants with gross blood in stools: a prospective, controlled study. Early Hum Dev (2014) 90(10):579-85. doi:10.1016/j. earlhumdev.2014.07.004

96. Torrazza RM, Ukhanova M, Wang X, Sharma R, Hudak ML, Neu J, et al. Intestinal microbial ecology and environmental factors affecting necrotizing enterocolitis. PLoS One (2013) 8(12):e83304. doi:10.1371/journal. pone. 0083304

97. Neu J. Probiotics and necrotizing enterocolitis. Clin Perinatol (2014) 41(4):967-78. doi:10.1016/j.clp.2014.08.014

98. Fituch CC, Palkowetz KH, Goldman AS, Schanler RJ. Concentrations of IL-10 in preterm human milk and in milk from mothers of infants with necrotizing enterocolitis. Acta Paediatr (2004) 93(11):1496-500. doi:10.1111/j. 1651-2227.2004.tb02636.x

99. Trend S, Strunk T, Lloyd ML, Kok CH, Metcalfe J, Geddes DT, et al. Levels of innate immune factors in preterm and term mothers' breast milk during the 1st month postpartum. Br J Nutr (2016) 115(7):1178-93. doi:10.1017/ S0007114516000234

100. Castellote C, Casillas R, Ramirez-Santana C, Perez-Cano FJ, Castell M, Moretones MG, et al. Premature delivery influences the immunological composition of colostrum and transitional and mature human milk. J Nutr (2011) 141(6):1181-7. doi:10.3945/jn.110.133652

101. Maheshwari A, Kelly DR, Nicola T, Ambalavanan N, Jain SK, Murphy-Ullrich J, et al. TGF-beta2 suppresses macrophage cytokine production and mucosal inflammatory responses in the developing intestine. Gastroenterology (2011) 140(1):242-53. doi:10.1053/j.gastro.2010.09.043

102. Wang XF, Cao RM, Li J, Wu J, Wu SM, Chen TX. Identification of sociodemographic and clinical factors associated with the levels of human beta-defensin- 1 and human beta-defensin- 2 in the human milk of Han Chinese. $\mathrm{Br}$ J Nutr (2014) 111(5):867-74. doi:10.1017/S0007114513003292 
103. Armogida SA, Yannaras NM, Melton AL, Srivastava MD. Identification and quantification of innate immune system mediators in human breast milk. Allergy Asthma Proc (2004) 25(5):297-304.

Conflict of Interest Statement: The authors declare that the research was conducted in the absence of any commercial or financial relationships that could be construed as a potential conflict of interest.
Copyright (c) 2017 Cacho and Lawrence. This is an open-access article distributed under the terms of the Creative Commons Attribution License (CC BY). The use, distribution or reproduction in other forums is permitted, provided the original author(s) or licensor are credited and that the original publication in this journal is cited, in accordance with accepted academic practice. No use, distribution or reproduction is permitted which does not comply with these terms. 